REVIEW ARTICLE

\title{
Candida parapsilosis Secreted Lipase as an Important Virulence Factor
}

\author{
Renata Toth, Adel Toth, Csaba Vagvölgyi and Attila Gacser ${ }^{*}$
}

Department of Microbiology, University of Szeged, Szeged, Hungary

\begin{tabular}{l} 
A R T I C L E H I S T O R Y \\
\hline Received: February 23, 2016 \\
Revised: July 15,2016 \\
Accepted: July 21,2016 \\
DOI: $10.2174 / 138920371766616081$ \\
3163054
\end{tabular}

Abstract: The prevalence of Candida parapsilosis, an opportunistic human pathogenic fungal species, is increasing at an alarming rate in the hospital environment. Patients at risk for C. parapsilosis infection include those with immunosuppression, such as individuals with cancer, AIDS, and low birth weight premature neonates as well as patients that had undergone abdominal surgery. Neonatal candidiasis caused by $C$. parapsilosis has been widely reported across the globe. Various reports have shown that, compared to other Candida species, certain C. parapsilosis clinical isolates were less susceptible to antifungals such as amphotericin B, fluconazole, and caspofungin. In addition, some studies have even reported multi-echinocandin or multi-azole resistant strains of C. parapsilosis. C. parapsilosis has several virulence factors that contribute to its capacity for host invasion and among these factors extracellular lipases have a major role in pathogenesis. In this review we have collected all the recent relevant studies that confirm the involvement of secreted lipases in C. parapsilosis pathogenesis, using both in vitro and in vivo models of infection. Of particular note, an available lipase deficient $C$. parapsilosis strain has been utilized to demonstrate that the lack of secreted lipases decreased virulence, reduced tissue damage, and was less able to survive within phagocytes or mice compared to the wild type. Since fungal secreted lipases have different characteristics than lipolytic enzymes present in humans, C. parapsilosis extracellular lipases may be potential targets for the development of novel antifungal drugs.

Keywords: Candida parapsilosis, extracellular lipase, pathogenicity, macrophages, dendritic cells, reconstituted human tissue, in vivo models.

\section{INTRODUCTION}

Despite of the wide range of available antifungal medicines, nosocomial fungal infections remain a major concern [1-3]. Recent studies detail that invasive candidiasis is the leading cause of mycosis-associated mortality worldwide [47]. Although the opportunistic pathogen Candida albicans is the most common species found in the hospital environment, numerous non-albicans Candida (NAC) species have been isolated from patients as a source of primary infection [8, 9]. More significantly, the frequency of invasive disease due to NAC species such as $C$. parapsilosis is rising alarmingly $[10,11]$. One reason for the increased incidence of $C$. parapsilosis infections is that the species displays notable resistance to antifungals drugs $[11,12]$. Several studies have reported C. parapsilosis clinical isolates with reduced susceptibility to different types of antifungals such as Amphotericin $\mathrm{B}$, fluconazole, anidulafungin, and caspofungin when compared to other Candida species [13-15, 14c]. In addition, multi-echinocandin and multi-azole-resistant clinical strains have been also isolated from patients [14b]. Horizontal transmission is also a characteristic of this species [16].

*Address correspondence to this author at the Department of Microbiology, University of Szeged, Közép fasor 52,H-7626 Szeged, Hungary;

Tel: +36/62544849; Fax:+36/62544823; E-mail: gacsera@gmail.com
Taken together, these factors suggest that, the number of resistant $C$. parapsilosis strains might further increase in the future.

Since the $90 \mathrm{~s}$, reports have frequently associated $C$. parapsilosis with nosocomial infections among children and newborns [17-21]. Several recent studies have further reported $C$. parapsilosis to be the predominant species causing neonatal infections at intensive care units (ICU), threatening especially low birth weight infants, with significant neonatal mortality [7, 22-24]. Additionally, diverse adult patients with diminished immunity are also at risk [25-28]. Further risk factors include the use of lipid rich parenteral nutrition and prolonged use of implanted devices, as $C$. parapsilosis readily forms and maintains stable biofilms on the surfaces of commonly used medical equipments [29].

Microbial secreted hydrolytic enzymes are known to contribute to the process of fungal pathogenesis. Such enzymes include extracellular proteinases, phospholipases and lipases [30-32]. The role of fungal secreted lipases is pleiotrop as they modulate the immune response in various ways [33]. Both cellular and humoral host responses are affected and they also contribute to host tissue damage, allowing fungal cells to invade the host [33-35]. Based on a large number of recent studies, $C$. parapsilosis is considered an emerging 
pathogen, and since its clinical relevance is clear, attention should be increased towards this NAC species.

A number of reports and previous reviews have already demonstrated the role of $C$. parapsilosis in nosocomial infections $[23,24,29,36]$. In this review, we aim to provide a description of a critically important effector protein of $C$. parapsilosis that has been proven to promote invasion, including altering host immune responses to the benefit of the fungus. We will detail how fungal secreted lipases influence the virulence of $C$. parapsilosis and thus provide evidence that fungal lipases should be considered a target for developing antifungals in the future.

\section{FUNCTION OF FUNGAL SECRETED LIPASES}

Fungal secreted lipases can have a variety of functions, due to their ability to catalyze a wide range of reactions such as the hydrolysis or synthesis of triacylglycerols [37]. Many microbial extracellular lipases are used for industrial purposes as biocatalysts and Candida species are frequently used to produce hydrolytic enzymes for industrial use [38, 39]. For example, C. antartica and C. rugosa secreted lipases are the most frequently used biocatalysts that are involved in detergent industry, biosensor modulation, and even biocatalytic resolution of pharmaceuticals such as antibiotics, virucids, probiotics and non-steroid anti-inflammatory drugs (NSAIDs) [38, 40, 41]. Thus, fungal secreted lipases are considered beneficial for biotechnological applications.

However, the role of secreted lipase as a potential virulence factor on the level of a pathogenic microorganism is also quite evident $[31,42]$. In addition to the key roles of lipases in nutrient acquisition, they might also augment adhesion to host tissues, lyse competitive microflora, and modulate host inflammatory reactions, which can also result in further tissue damage [29, 43]. Therefore, fungal secreted lipases contribute to host invasion in diverse ways.

C. albicans is known to have 10 lipase encoding genes, one (LIP1) characterized by Fu et al. [44] and nine (LIP2LIP10) by Hube et al. [31]. Further examinations of C. albicans lipases revealed that individual genes are regulated differently during an infection and gene expression of each ORF was mainly dependent upon the stage of infection [45]. Interestingly, the different genes had variable contributions to virulence. The role of LIP5 and LIP8 in C. albicans virulence was highlighted as their expression levels remained the highest when examining isolates recovered from systemically infected mice or from human oral specimens [45]. Furthermore, the generation of a C. albicans strain lacking LIP8 led to experiments demonstrating $\bigcirc$ this null mutant was less virulent in vivo compared to wrutype [46]. These reports suggest that differential regulation of lipase gene expression has helped C. albicans to adapt to the human host.

Lipid acquisition from the host has already been reported in Candida species. Both $C$. albicans and C. parapsilosis assimilate exogenic arachidonic acid in order to produce human prostaglandin-like molecules $\left(\mathrm{PGE}_{2}\right.$ and $\left.\mathrm{PGD}_{2}\right)[47$, 48]. Fungal prostaglandins were shown to modulate host immune responses via a variety of mechanisms to promote host colonization [49]. Furthermore, C. albicans morphogenesis is also influenced by $\mathrm{PGE}_{2}$ [49]. Secreted lipases contribute to the release of lipid intermediers during host invasion, and therefore also indirectly influence fungal virulence.

CpFIT2 (Fat storage-Inducing Transmembrane protein 2) is involved in fungal lipid droplet (LD) formation. According to Nguyen et al. FIT2 has a role in triacil glycerol (TAG) synthesis and may be required for the balance between lipid species via LD formation. It is notable, that defect of CPFIT2 lead $D$ educed LD formation, altered fatty acid composition 2 th disabilities and increased susceptibility to oxidative stressor. Furthermore, FIT2 deletion also resulted in reduced virulence in murine infection models, further supporting the role of fungal lipids in pathogenicity [50].

C. parapsilosis is considered to be less pathogenic than C. albicans, although its relevance is increasing in the hospital environment and it even outranks C. albicans in some health care centers as a cause of invasive candidiasis [29]. $C$. parapsilosis has only two indentified secreted lipase coding genes [29]. The presence of only two secreted lipase encoding genes facilitated the construction of a secreted lipase-null strain, a double knock-out strain, which has proven to be a valuable tool for studying the role of extracellular lipases during host-pathogen interactions.

\section{IDENTIFICATION OF $C$. PARAPSILOSIS SECRETED LIPASES}

$\mathrm{Fu}$ et al. published the first report that suggested the presence of secreted lipases in C. parapsilosis in 1997 [44]. Five years later, Neugnot et al. identified two lipase encoding ORFs in this species [51]. Amino acid sequence comparison revealed that these identified proteins might belong to the same Candida lipase gene family that was described by Hube et al. in C. albicans, thus they might be also involved in fungal pathogenesis. The newly found $C$. parapsilosis lipase encoding ORFs showed the highest homology with $C$. albicans LIP1. Accordingly, the ORFs were named $C p L I P 1$ and $C p L I P 2$. Expression analysis of the two $C$. parapsilosis lipase genes in $S$. cerevisiae and later in $P$. pastoris revealed that only $C p L I P 2$ coded for a functional protein $[51,52]$. C. parapsilosis LIP1 and LIP2 are encoded by two neighboring ORF regions with an approximate size of $1300 \mathrm{bp}$ for each [51]. Both lipases consist of a deduced 465 amino acid sequence with two CUG codons present in CpLIP1 but none in CPLIP2. As C . parapsilosis is a member of the CUG-clade (serine instead of leucine translation), this might be an explanation why the enzyme encoded by $C p L I P 1$ is not functional when expressed in $S$. cerevisiae [51]. CpLIP2 was further described with a molecular mass of $60 \mathrm{kDa}$ and was shown to contain the Gly-X-Ser-X-Gly consensus motif that is highly conserved and commonly found in the serine catalytic core of lipolytic enzymes [51]. The lipase from $C$. parapsilosis catalyses the alcoholysis of various esters. According to Briand el al., this lipase showed the highest activity when unsaturated fatty acids with a cis-delta 9 double bond or the ester bond of long-chain fatty acids were present in the medium [53]. During the investigation of biochemical properties, it was also shown that the catalytic activity was also dependent upon the temperature of the reactant medium [53]. 
After whole genome sequencing, two additional LIP-like genes were suggested that may be present in the C. parapsilosis genome, however, these remained uninvestigated [29]. Expression studies of $C p L I P$ genes using other yeasts highly contributed to our knowledge on C. parapsilosis lipase secretion, but did not address their role in virulence. Although there were advances in C. parapsilosis genetic manipulation $[12,54]$, it was not until 2007 that an innovative method for targeted gene deletion became available, and LIP1 - LIP2 were one of the first genes to be deleted from the diploid genome of $C$. parapsilosis, using a dominant selection marker carrier flipper cassette [34]. The available null mu-

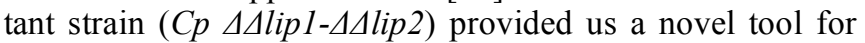
studying $C$. parapsilosis lipases and facilitated numerous subsequent virulence related investigations. Early studies demonstrated the role of CPLIP1-LIP2 in viability in lipid rich environment, biofilm formation, and adherence to host surfaces as well as to immune response modulation [34].

\section{IN VITRO HOST EFFECTOR CELL - C. PARAPSILO- SIS INTERACTIONS}

Using the previously mentioned lipase deficient strain, several studies confirmed the role of $C$. parapsilosis lipases in virulence (Fig. 1) [33, 34, 55, 56]. Initial studies focused on the interactions of $C$. parapsilosis with murine macro-

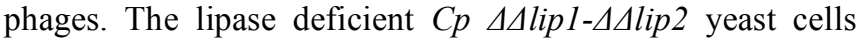
induced higher phagocytic and killing efficiency by murine macrophages in vitro when compared to the lipase secreting wild type cells (Fig. 2) [34]. Subsequent studies with human peripheral blood mononuclear cell - derived macrophages (PBMC-DMs) [33] and dendritic cells (DCs) [55] reported

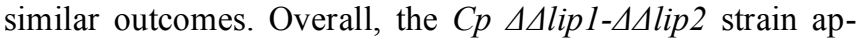
peared to be less virulent, as both types of human primary cells were able to kill mutant cells in a higher ratio compared to wild type cells. Intracellular survival of $C$. parapsilosis cells following phagocytosis has been recently reported [35]. In contrast with DCs, no difference was detected in the phagocytic efficiency of fungal cells by PBMC-DMs, although early phago-lysosome fusion was detected in both cases $[33,55]$. These results led to the conclusion that fungal lipase secretion delays phagosome maturation, thus is considered to be one of the factors that contribute to fungal survival inside host cells.

In addition to their roles as professional phagocytes and professional antigen presenting cells, PBMC-DMs and DCs release certain types of effector molecules or cytokines to modulate immune responses. These cytokines include interleukin -1 (IL-1), interleukin - 6 (IL-6) and tumor necrosis factor alpha $(\mathrm{TNF} \alpha)$ as major inducers of inflammatory reactions; IL-8 as a neutrophil chemotactic factor and other chemokines to recruit leukocytes to the infection site [57, 58]. Cyclooxygenase $2(\mathrm{COX}-2)$ is essential for the synthesis of the biologically active inflammation inducer prostaglandin E2 [48]. Toth A. et al. and Nagy and Filkor et al. reported that both DCs and PBMC-DMs released IL-1, IL-6 and $\mathrm{TNF} \alpha$ pro-inflammatory cytokines at a markedly increased level in the presence $C p \Delta \Delta l i p 1-\Delta \Delta l i p 2$ cells compared to that occurring in the presence of wild type yeast cells and an elevated level of IL-8 was detected after DCs were challenged with $C p \Delta \Delta l i p 1-\Delta \Delta l i p 2$ compared to wild type cells [55]. Similarly, higher levels of IL-8 and COX-2 expression were observed when PBMC-DMs were treated with the lipase deficient mutant in comparison with wild type strain. Interestingly, the anti-inflammatory response of PBMC-DM was also modulated, as an elevated IL-10 level was found

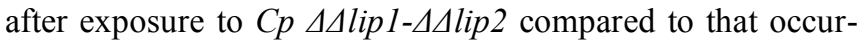
ring after challenge with wild type yeast $[33,55]$.

In summary, the lack of the secreted lipase led to a stronger inflammatory response, shown by the increase in the pro-inflammatory cytokine and chemokine expression levels, as well as by more efficient killing and phagocytosis of $C p$

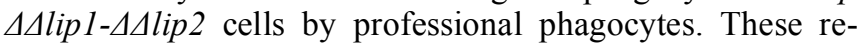
ports suggest that $C$. parapsilosis secreted lipases suppress both the cellular and humoral immune responses and thus have a protective role against host responses.

\section{TISSUE MODELS FOR C. PARAPSILOSIS EX- TRACELLULAR LIPASES}

In the macrophage - C. parapsilosis model, host cell damage by fungal cells was not affected by the presence of secreted lipases [33]. However, this did not mean that the extracellular lipases could not impact more complex tissues and the utilization of reconstituted human tissue (RHT) models allowed for the first assessment of $C$. parapsilosis lipase on virulence. Although RHTs had been used to model cutaneous, oral and vaginal C. albicans disease [59-61], they had not been applied to the study of C. parapsilosis to assess the histopathological effect of microbial secreted hydrolytic enzymes such as fungal extracellular lipases [34, 62]. In lipid rich environment fungi use secreted lipases to digest lipids in order to supply basic nutrient needs [29]. Oral epithelial RHT challenged with C. parapsilosis wild type cells develop severe histopathological changes such as atrophy, edema, and clefting in all tissue layers, along with higher rates of

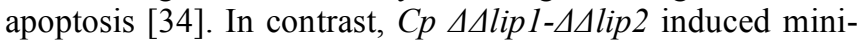
mal injury, as the infected tissue was histologically similar to the uninfected control and their ability to attach to the epithelial surface was significantly decreased compared to wild type [34]. Using lactate-dehydrogenase (LDH) release as a determinant of RHT damage, the wild type $C$. parapsilosis yeasts caused significantly reduced cellular damage compared to the lipase mutant cells [34]. Additionally, inhibition of lipase by Ebelactone B, quinine or acetylysalicylic acid abrogated RHT infection and damage caused by C. parapsilosis $[62,63]$. These findings further supported the involvement of $C$. parapsilosis extracellular lipases in tissue adhesion and destructive disease.

\section{IN VIVO INFECTION MODELS TO STUDY $C$. PARAPSILOSIS LIPASE FUNCTION}

In order to study systemic immune responses and inflammation triggered by pathogenic fungi, numerous in vivo infection models are in use. Even though, newly emerging non-conventional in vivo models of infection are becoming popular, mammalian models are still of central importance $[64,65]$. Although Galleria mellonella has been described as a model to study Candida species virulence, including $C$. parapsilosis $[65,66]$, mice remain the most suitable and widely accepted model of candidiasis; however, occasionally, certain types of rats are also used. A common method to investigate non-lethal disease is to perform fungal burden 


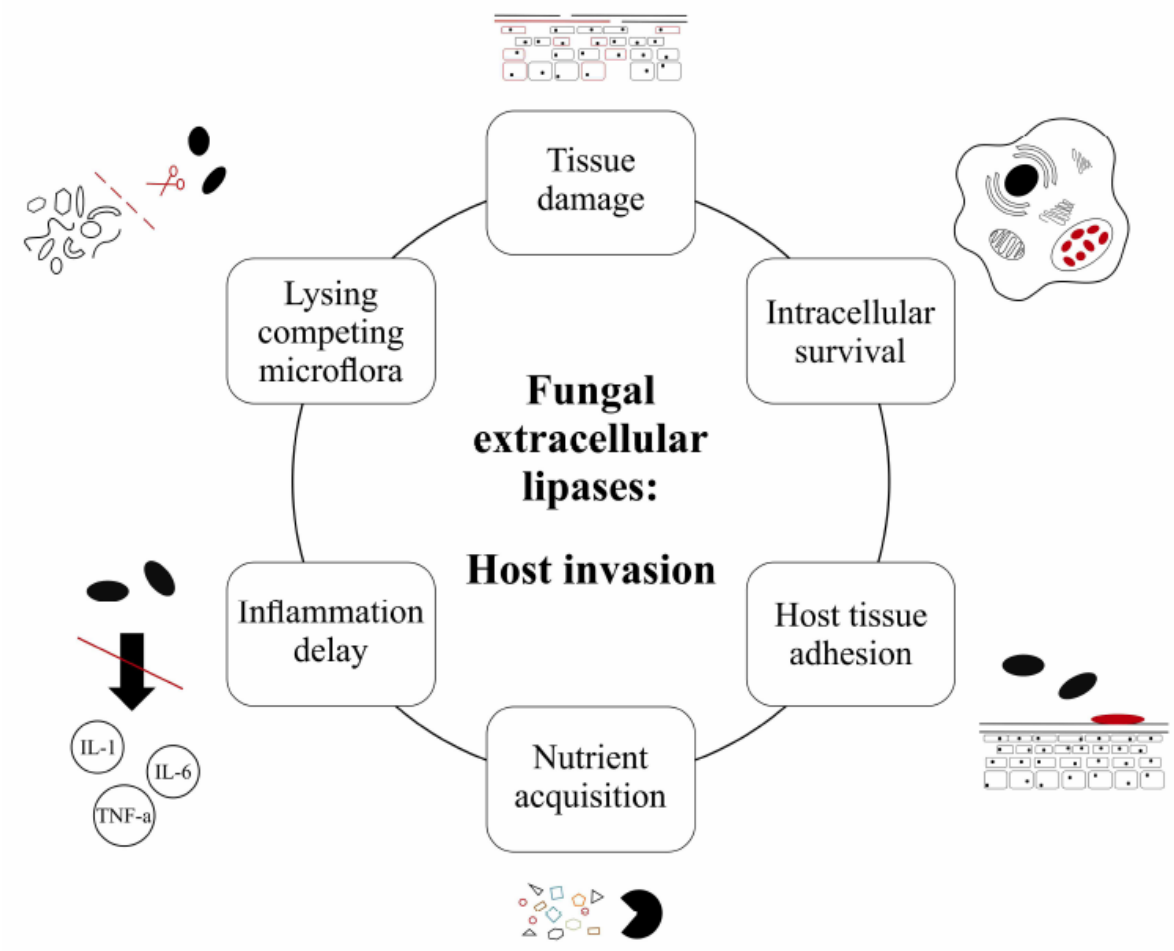

Fig. (1). The role of fungal extracellular lipases in host invasion. The image represents the putative role of $C$. parapsilosis secreted lipases during pathogenesis. Fungal secreted lipases directly contribute to nutrient acquisition and host cell lysis. Microbial extracellular lipases may also be responsible for directly lysing competitive microflora. Besides their direct roles in virulence they might influence fungal adhesion properties; delay inflammatory processes due to the release of chemical mediators from damaged cells; and contribute to intracellular survival as a result of phago-lysosome fusion inhibition.
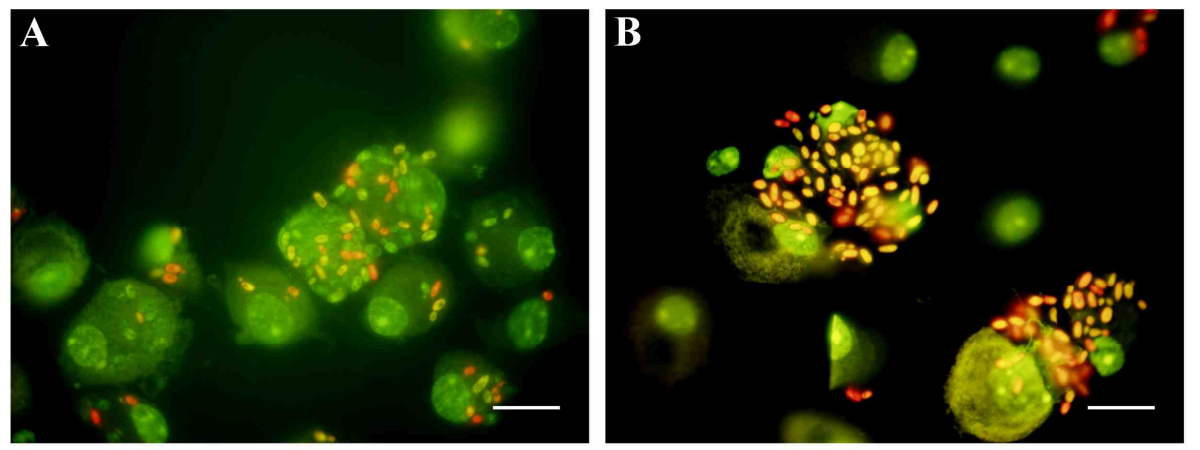

Fig. (2). Phagocytosis of $\boldsymbol{C}$. parapsilosis cells. Representative image shows the phagocytosis of $C$. parapsilosis wild type (A) and lipase null mutant cells (B) by human peripheral blood mononuclear cell - derived macrophages. Acridine orange and crystal violet were used for staining. Orange / red fluorescence indicates cell death, while live cells emit green fluorescence. Scale bar: $20 \mu \mathrm{m}$.

assessments of organs by means of colony forming unit (CFU) counting of plated organ homogenates. Using a intraperitoneal murine infection model, it has been demonstrated that $C p \Delta \Delta$ lip1- $\Delta \Delta$ lip2 cells were able to accumulate less in the kidney, liver and spleen of BALB/c mices 2 days post infection [34], but these mutant cells were eradicated within 4 days after the infection, which was significantly faster than clearance of wild type yeast [34]. As C. parapsilosis is one of the leading species associated with neonatal invasive infections, efforts have been applied to developing a suitable model to mimic newborn-related candidiasis [6769]. Using newborn Sprague-Dawley rats, Trofa et al. demonstrated that premature neonates were highly susceptible to candidal infections. Moreover, this rat pup cancruar infections. Moreover, this rat pup model proved to be effective for detecting the effects of the presence or absence of extracellular lipases. In their experiments, they infected newborn rats intravenously (IV), intragastrically (IG) and intraperitoneally (IP) either with the lipase secretion deficient strain of $C$. parapsilosis, a LIP\& deficient strain of C. albicans or their corresponding wild type strains. Notably,

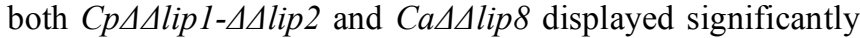
lower virulence compared to wild type strains as indicated by reduced organ fungal burdens. These findings of in vivo studies further demonstrate the crucial role of fungal secreted lipases in C. parapsilosis pathogenicity. 


\section{CONCLUSIONS}

C. parapsilosis is an opportunistic human pathogenic species that is commonly associated with nosocomial fungal diseases, and this species continues to emerge in incidence and importance. Fundamental molecular investigations coupled with in vitro and in vivo studies have made major strides in elucidating several of the factors that facilitate the pathogenesis of $C$. parapsilosis. One of the best described virulence factors is the secreted lipases of $C$. parapsilosis, and the availability of lipase deletion strains has facilitated the study of $C$. parapsilosis virulence properties. There are now numerous reports that clearly detail the importance of $C$. parapsilosis extracellular lipases in pathogenesis as their presence alters cellular and humoral immune responses, delays inflammatory reactions, contributes to host tissue adhesion and promotes intracellular survival. In this review, we described the key role of secreted lipases in $C$. parapsilosis virulence, as another potential factor of this species that can be targeted by a novel class of antifungals of future medicine.

\section{LIST OF ABBREVIATIONS}

\begin{tabular}{|c|c|c|}
\hline Cp $\Delta \Delta l i p 1-\Delta \Delta l i p 2$ & $=$ & $\begin{array}{l}\text { C. parapsilosis LIP1-LIP2 secreted } \\
\text { lipase homozygous deletion strain; }\end{array}$ \\
\hline PBMC-DM & $=$ & $\begin{array}{l}\text { peripheral blood mononuclear cell } \\
\text { - derived macrophages; }\end{array}$ \\
\hline DC & & dendritic cell; \\
\hline RHT & & reconstituted human tissue. \\
\hline
\end{tabular}

\section{CONFLICT OF INTEREST}

The author(s) confirm that this article content has no conflict of interest.

\section{ACKNOWLEDGEMENTS}

RT's research was supported by the European Union and the State of Hungary, co-financed by the European Social Fund in the framework of TÁMOP 4.2.4. A/2-11-1-20120001 'National Excellence Program'. AG is supported by OTKA NN113153 and by NF84006. AG is further supported by the János Bolyai Research Scholarship of the Hungarian Academy of Sciences. This work was supported by the project GINOP-2.3.3-15-2016-00006.

\section{REFERENCES}

[1] Lepak, A.; Andes, D. Fungal sepsis: optimizing antifungal therapy in the critical care setting. Crit. Care Clin., 2011, 27(1), 123-147.

[2] Jain, A.; Jain, S.; Rawat, S. Emerging fungal infections among children: A review on its clinical manifestations, diagnosis, and prevention. J. Pharm. Bioallied Sci., 2010, 2(4), 314-320.

[3] Low, C.Y.; Rotstein, C. Emerging fungal infections in immunocompromised patients. F1000 Med. Rep., 2011, 3, 14.

[4] Pfaller, M.A.; Diekema, D.J. Epidemiology of invasive candidiasis: a persistent public health problem. Clin. Microbiol. Rev., 2007, 20(1), 133-163.

[5] Caggiano, G.; Coretti, C.; Bartolomeo, N.; Lovero, G.; De Giglio, O.; Montagna, M.T. Candida Bloodstream Infections in Italy: Changing Epidemiology during 16 Years of Surveillance. BioMed. Res. Int., 2015, 2015, 256580.

[6] Ruan, S.Y.; Hsueh, P.R. Invasive candidiasis: an overview from Taiwan. J. Formos. Med. Assoc., 2009, 108(6), 443-451.
[19] Saiman, L.; Ludington, E.; Dawson, J.D.; Patterson, J.E.; RangelFrausto, S.; Wiblin, R.T.; Blumberg, H.M.; Pfaller, M.; Rinaldi, M.; Edwards, J.E.; Wenzel, R.P.; Jarvis, W.; National Epidemiology of Mycoses Study, G. Risk factors for Candida species colonization of neonatal intensive care unit patients. Pediatr. Infect. Dis. J., 2001, 20(12), 1119-1124.

[20] el-Mohandes, A.E.; Johnson-Robbins, L.; Keiser, J.F.; Simmens, S.J.; Aure, M.V. Incidence of Candida parapsilosis colonization in an intensive care nursery population and its association with invasive fungal disease. Pediatr. Infect. Dis. J., 1994, 13(6), 520-524.

[21] Bendel, C.M. Colonization and epithelial adhesion in the pathogenesis of neonatal candidiasis. Semin. Perinatol., 2003, 27(5), 357-364.

[22] Clerihew, L.; Lamagni, T.L.; Brocklehurst, P.; McGuire, W. Candida parapsilosis infection in very low birthweight infants. Arch. Dis. Child Fetal Neonatal Ed., 2007, 92(2), F127- F129.

[23] Pammi, M.; Holland, L.; Butler, G.; Gacser, A.; Bliss, J.M. Candida parapsilosis is a significant neonatal pathogen: a systematic review and meta-analysis. Pediatr. Infect. Dis. J., 2013, 32(5), e206- e216.

[24] Chow, B.D.; Linden, J.R.; Bliss, J.M. Candida parapsilosis and the neonate: epidemiology, virulence and host defense in a unique patient setting. Expert. Rev. Anti Infect. Ther., 2012, 10(8), 935-946.

[25] Torres, H.A.; Kontoyiannis, D.P.; Rolston, K.V. High-dose fluconazole therapy for cancer patients with solid tumors and can- 
didemia: an observational, noncomparative retrospective study. Support Care Cancer, 2004, 12(7), 511-516.

[26] Patel, R.; Grogg, K.L.; Edwards, W.D.; Wright, A.J.; Schwenk, N.M. Death from inappropriate therapy for Lyme disease. Clin. Infect. Dis., 2000, 31(4), 1107-1109.

[27] Almirante, B.; Rodriguez, D.; Cuenca-Estrella, M.; Almela, M.; Sanchez, F.; Ayats, J.; Alonso-Tarres, C.; Rodriguez-Tudela, J.L.; Pahissa, A. Epidemiology, risk factors, and prognosis of Candida parapsilosis bloodstream infections: case-control population-based surveillance study of patients in Barcelona, Spain, from 2002 to 2003. J. Clin. Microbiol., 2006, 44(5), 1681-1685.

[28] Brito, L.R.; Guimaraes, T.; Nucci, M.; Rosas, R.C.; Paula Almeida, L.; Da Matta, D.A.; Colombo, A.L. Clinical and microbiological aspects of candidemia due to Candida parapsilosis in Brazilian tertiary care hospitals. Med. Mycol., 2006, 44(3), 261-266.

[29] Trofa, D.; Gacser, A.; Nosanchuk, J.D. Candida parapsilosis, an emerging fungal pathogen. Clin. Microbiol. Rev., 2008, 21(4), 606625 .

[30] Horvath, P.; Nosanchuk, J.D.; Hamari, Z.; Vagvolgyi, C.; Gacser, A. The identification of gene duplication and the role of secreted aspartyl proteinase 1 in Candida parapsilosis virulence. $J$. Infect. Dis., 2012, 205(6), 923-933.

[31] Hube, B.; Stehr, F.; Bossenz, M.; Mazur, A.; Kretschmar, M.; Schafer, W. Secreted lipases of Candida albicans: cloning, characterisation and expression analysis of a new gene family with at least ten members. Arch. Microbiol., 2000, 174(5), 362-374.

[32] Ghannoum, M.A. Potential role of phospholipases in virulence and fungal pathogenesis. Clin. Microbiol. Rev., 2000, 13(1), 122-143, table of contents.

[33] Toth, A.; Nemeth, T.; Csonka, K.; Horvath, P.; Vagvolgyi, C.; Vizler, C.; Nosanchuk, J.D.; Gacser, A. Secreted Candida parapsilosis lipase modulates the immune response of primary human macrophages. Virulence, 2014, 5(4), 555-562.

[34] Gacser, A.; Trofa, D.; Schafer, W.; Nosanchuk, J.D. Targeted gene deletion in Candida parapsilosis demonstrates the role of secreted lipase in virulence. J. Clin. Invest., 2007, $117(10), 3049-3058$.

[35] Toth, R.; Toth, A.; Papp, C.; Jankovics, F.; Vagvolgyi, C.; Alonso, M.F.; Bain, J.M.; Erwig, L.P.; Gacser, A. Kinetic studies of Candida parapsilosis phagocytosis by macrophages and detection of intracellular survival mechanisms. Front. Microbiol., 2014, 5, 633.

[36] van Asbeck, E.C.; Clemons, K.V.; Stevens, D.A. Candida parapsilosis: a review of its epidemiology, pathogenesis, clinical aspects, typing and antimicrobial susceptibility. Crit. Rev. Microbiol., 2009, 35(4), 283-309.

[37] Brockerhoff, H. Model of interaction of polar lipids, cholesterol, and proteins in biological membranes. Lipids, 1974, 9(9), 645-650.

[38] Liu, Z.Q.; Zheng, X.B.; Zhang, S.P.; Zheng, Y.G. Cloning, expression and characterization of a lipase gene from the Candida antarctica ZJB09193 and its application in biosynthesis of vitamin A esters. Microbiol. Res., 2012, 167(8), 452-460.

[39] Tang, S.J.; Shaw, J.F.; Sun, K.H.; Sun, G.H.; Chang, T.Y.; Lin, C.K.; Lo, Y.C.; Lee, G.C. Recombinant expression and characterization of the Candida rugosa lip4 lipase in Pichia pastoris: comparison of glycosylation, activity, and stability. Arch. Biochem. Biophys., 2001, 387(1), 93-98.

[40] Dominguez de Maria, P.; Sanchez-Montero, J.M.; Sinisterra, J.V.; Alcantara, A.R. Understanding Candida rugosa lipases: an overview. Biotechnol. Adv., 2006, 24(2), 180-196.

[41] Benjamin, S.; Pandey, A. Candida rugosa lipases: molecular biology and versatility in biotechnology. Yeast, 1998, 14(12), 10691087.

[42] Bramono, K.; Yamazaki, M.; Tsuboi, R.; Ogawa, H. Comparison of proteinase, lipase and alpha-glucosidase activities from the clinical isolates of Candida species. Jpn. J. Infect. Dis., 2006, 59(2), 7376

[43] Nguyen, L.N.; Gacser, A.; Nosanchuk, J.D. Secreted lipases supply fatty acids for yeast growth in the absence of de novo fatty acid synthesis. Virulence, 2011, 2(6), 538-541.

[44] Fu, Y.; Ibrahim, A.S.; Fonzi, W.; Zhou, X.; Ramos, C.F.; Ghannoum, M.A. Cloning and characterization of a gene (LIP1) which encodes a lipase from the pathogenic yeast Candida albicans. Microbiology, 1997, 143(Pt 2), 331-340.

[45] Stehr, F.; Felk, A.; Gacser, A.; Kretschmar, M.; Mahnss, B.; Neuber, K.; Hube, B.; Schafer, W. Expression analysis of the Candida albicans lipase gene family during experimental infections and in patient samples. FEMS Yeast Res., 2004, 4(4-5), 401-408.
[46] Gacser, A.; Stehr, F.; Kroger, C.; Kredics, L.; Schafer, W.; Nosanchuk, J.D. Lipase 8 affects the pathogenesis of Candida albicans. Infect. Immun., 2007, 75(10), 4710-4718.

[47] Noverr, M.C.; Toews, G.B.; Huffnagle, G.B. Production of prostaglandins and leukotrienes by pathogenic fungi. Infect. Immun., 2002, 70(1), 400-402.

[48] Grozer, Z.; Toth, A.; Toth, R.; Kecskemeti, A.; Vagvolgyi, C; Nosanchuk, J.D.; Szekeres, A.; Gacser, A. Candida parapsilosis produces prostaglandins from exogenous arachidonic acid and OLE2 is not required for their synthesis. Virulence, 2015, 6(1), 8592.

[49] Noverr, M.C.; Phare, S.M.; Toews, G.B.; Coffey, M.J.; Huffnagle, G.B. Pathogenic yeasts Cryptococcus neoformans and Candida albicans produce immunomodulatory prostaglandins. Infect. Immun. 2001, 69(5), 2957-2963.

[50] Nguyen, L.N.; Hamari, Z.; Kadereit, B.; Trofa, D.; Agovino, M.; Martinez, L.R.; Gacser, A.; Silver, D.L.; Nosanchuk, J.D. Candida parapsilosis fat storage-inducing transmembrane (FIT) protein 2 regulates lipid droplet formation and impacts virulence. Microbes Infect., 2011, 13(7), 663-672.

[51] Neugnot, V.; Moulin, G.; Dubreucq, E.; Bigey, F. The lipase/acyltransferase from Candida parapsilosis: molecular cloning and characterization of purified recombinant enzymes. Eur. J. Biochem., 2002, 269(6), 1734-1745.

[52] Brunel, L.; Neugnot, V.; Landucci, L.; Boze, H.; Moulin, G.; Bigey, F.; Dubreucq, E. High-level expression of Candida parapsilosis lipase/acyltransferase in Pichia pastoris. J. Biotechnol., 2004, 111(1), 41-50.

[53] Briand, D.; Dubreucq, E.; Grimaud, J.; Galzy, P. Substrate specificity of the lipase from Candida parapsilosis. Lipids, 1995, 30(8), 747-754

[54] Nosek, J.; Adamikova, L.; Zemanova, J.; Tomaska, L.; Zufferey, R.; Mamoun, C.B. Genetic manipulation of the pathogenic yeast Candida parapsilosis. Curr. Genet., 2002, 42(1), 27-35.

[55] Nagy, I.; Filkor, K.; Nemeth, T.; Hamari, Z.; Vagvolgyi, C.; Gacser, A. In vitro interactions of Candida parapsilosis wild type and lipase deficient mutants with human monocyte derived dendritic cells. BMC Microbiol., 2011, 11, 122 .

[56] Trofa, D.; Soghier, L.; Long, C.; Nosanchuk, J.D.; Gacser, A ; Goldman, D.L. A rat model of neonatal candidiasis demonstrates the importance of lipases as virulence factors for Candida albicans and Candida parapsilosis. Mycopathologia, 2011, 172(3), 169-178.

[57] de Oliveira, S.; Reyes-Aldasoro, C.C.; Candel, S.; Renshaw, S.A.; Mulero, V.; Calado, A. Cxc18 (IL-8) mediates neutrophil recruitment and behavior in the zebrafish inflammatory response. $\mathrm{J}$. Immunol., 2013, 190(8), 4349-4359.

[58] Mizel, S.B. The interleukins. FASEB J., 1989, 3(12), 2379-2388.

[59] Korting, H.C.; Patzak, U.; Schaller, M.; Maibach, H.I. A model of human cutaneous candidosis based on reconstructed human epidermis for the light and electron microscopic study of pathogenesis and treatment. J. Infect., 1998, 36(3), 259-267.

[60] Schaller, M.; Zakikhany, K.; Naglik, J.R.; Weindl, G.; Hube, B. Models of oral and vaginal candidiasis based on in vitro reconstituted human epithelia. Nat. Protocols, 2006, 1(6), 2767-2773.

[61] Bernhardt, J.; Herman, D.; Sheridan, M.; Calderone, R. Adherence and invasion studies of Candida albicans strains, using in vitro models of esophageal candidiasis. J. Infect. Dis., 2001, 184(9), 1170-1175.

[62] Gacser, A.; Schafer, W.; Nosanchuk, J.S.; Salomon, S.; Nosanchuk, J.D. Virulence of Candida parapsilosis, Candida orthopsilosis, and Candida metapsilosis in reconstituted human tissue models. Fungal Genet. Biol., 2007, 44(12), 1336-1341.

[63] Trofa, D.; Agovino, M.; Stehr, F.; Schafer, W.; Rykunov, D.; Fiser, A.; Hamari, Z.; Nosanchuk, J.D.; Gacser, A. Acetylsalicylic acid (aspirin) reduces damage to reconstituted human tissues infected with Candida species by inhibiting extracellular fungal lipases. Microbes Infect., 2009, 11(14-15), 1131-1139.

[64] Davis, M.M.; Alvarez, F.J.; Ryman, K.; Holm, A.A.; Ljungdahl, P.O.; Engstrom, Y. Wild-type Drosophila melanogaster as a model host to analyze nitrogen source dependent virulence of Candida albicans. PloS One, 2011, 6(11), e27434.

[65] Jacobsen, I.D. Galleria mellonella as a model host to study virulence of Candida. Virulence, 2014, 5(2), 237-239.

[66] Gago, S.; Garcia-Rodas, R.; Cuesta, I.; Mellado, E.; AlastrueyIzquierdo, A. Candida parapsilosis, Candida orthopsilosis, and 
Candida metapsilosis virulence in the non-conventional host Galleria mellonella. Virulence, 2014, 5(2), 278-285.

[67] Zaoutis, T.E.; Greves, H.M.; Lautenbach, E.; Bilker, W.B.; Coffin, S.E. Risk factors for disseminated candidiasis in children with candidemia. Pediatr. Infect. Dis. J., 2004, 23(7), 635-641.

[68] Fridkin, S.K.; Kaufman, D.; Edwards, J.R.; Shetty, S.; Horan, T. Changing incidence of Candida bloodstream infections among
NICU patients in the United States: 1995-2004. Pediatrics, 2006, 117(5), 1680-1687.

[69] Abelson, J.A.; Moore, T.; Bruckner, D.; Deville, J.; Nielsen, K. Frequency of fungemia in hospitalized pediatric inpatients over 11 years at a tertiary care institution. Pediatrics, 2005, 116(1), 61-67.

DISCLAIMER: The above article has been published in Epub (ahead of print) on the basis of the materials provided by the author. The Editorial Department reserves the right to make minor modifications for further improvement of the manuscript. 